\title{
Super-Fast Fabrication of MXene Film Through a Combination of Ion Induced Gelation and Vacuum-Assisted Filtration
}

\author{
Jingfeng Wang, ${ }^{1}$ Hui Kang, ${ }^{1}$ Haoxiang Ma, ${ }^{2}$ Yuyan Liu, ${ }^{1}$ Zhimin Xie, ${ }^{3}$ Youshan Wang ${ }^{3}$ and Zhimin Fan ${ }^{1, *}$
}

\begin{abstract}
MXene $\left(\mathrm{Ti}_{3} \mathrm{C}_{2} \mathrm{~T}_{x}\right)$ film produced through regular vacuum-assisted filtration have advantages of high conductivity, excellent mechanical performance, good surface wettability and others. However, the production method costs a large amount of time and energy, greatly limiting large-scale application of such film. Inspired by filtration of silt, we reveal and verify the feasibility of fast film forming through vacuum-assisted filtration of two-dimensional (2D) materials by using ions to induce MXene colloidal dispersion to transform into three-dimensional (3D) microgel in this work. A suitable gap between MXene microgel is the premise of sustainable fast water molecule filtration; the strong gelation and internal riveting effect inside the microgel ensure the formability of MXene film. Therefore, the production of MXene film is dramatically shortened from a few hours to just dozens of seconds. More importantly, MXene film prepared in this way can be folded and rubbed, and presents more excellent mechanical property, high electrochemical performance, and good electromagnetic interference shielding effective. This work provides theoretical and technical support for effective production of MXene film with excellent comprehensive properties and is of great significance to large-scale MXene film preparation and application.
\end{abstract}

Keywords: $\mathrm{Ti}_{3} \mathrm{C}_{2} \mathrm{~T}_{x}$ MXene film; Microgel; Super-fast preparation; Good electrical conductivity; High volumetric capacitance.

Received: 15 February 2021; Accepted date: 28 March 2021.

Article type: Research article.

\section{Introduction}

$\mathrm{Ti}_{3} \mathrm{C}_{2} \mathrm{~T}_{x}$ MXene ( $\mathrm{T}_{x}$ represents terminal functional groups, such as $-\mathrm{OH},-\mathrm{O}$ and $-\mathrm{F}$ ) is the most widely studied MXene, ${ }^{[1-}$ 3] which has the combination of extraordinary electrical conductivity, ${ }^{[4]}$ hydrophilicity ${ }^{[5]}$ and abundant surface chemically activity. ${ }^{[6]}$ These unique physical and chemical characteristics endow $\mathrm{Ti}_{3} \mathrm{C}_{2} \mathrm{~T}_{x}$ with outstanding versatility, designability and processability. Note that lately Gogotsi's work has confirmed that MXene $\left(\mathrm{Ti}_{3} \mathrm{C}_{2} \mathrm{~T}_{x}\right)$ production can be

${ }^{1}$ MIIT Key Laboratory of Critical Materials Technology for New Energy Conversion and Storage, School of Chemistry and Chemical Engineering, Harbin Institute of Technology, Harbin, Heilongjiang 150001, P. R. China.

${ }^{2}$ Deep Sea Engineering Division, Institute of Deep Sea Science and Engineering, Chinese Academy of Sciences, Sanya, Hainan 572000, P. R. China.

${ }^{3}$ National Key Laboratory of Science and Technology on Advanced Composites in Special Environments, Harbin Institute of Technology, Harbin, Heilongjiang 150080, P. R. China.

*E-mail: fanzm@hit.edu.cn (Z. Fan) expanded to industrial scale safely, easily and economically, with the properties practically the same as those produced in laboratories. ${ }^{[7]}$ This means that MXene is one of the few 2D materials that can potentially go from laboratories to industrial application.

Vacuum-assisted filtration is a regular technique widely used to separate two-dimensional (2D) material flakes from fluids to construct various 2D materials-based macroscopic assemblies. It is noteworthy that most of MXene flakes in MXene colloidal dispersion are individual and delaminated, under the effect of the vacuum pump, MXene flakes tightly deposit on the filter membrane surface face to face in a stretched manner, and freestanding MXene film with high intensity and outstanding conductivity can be obtained after drying. At present, MXene film fabricated through vacuumassisted filtration shows great potential for applications in fields such as electrochemical energy storage, ${ }^{[8-10]}$ electromagnetic interference shielding, ${ }^{[11,12]}$ smart materials ${ }^{[13-}$ ${ }^{16]}$ and other areas. ${ }^{[17,18]}$ However, this technique has obvious processing limitations: requiring a large amount of time and energy, especially for thick MXene film. The main reason is that once the filtration starts, some MXene flakes will quickly cover the filter membrane pore surface and automatically form 
film face-to-face in a tight accumulation manner; the remaining MXene flakes in the colloidal dispersion will stack layer by layer over the previously formed MXene film surface. As 2D materials normally show a high aspect ratio and barrier effect, ${ }^{[19-21]}$ water molecules need to move flow along the edges of MXene flakes, which thus significantly increases transport paths and difficulty in passing of water molecules. More importantly, extremely slow filtration will cause long time contact between MXene and oxygen-containing water, and then there is the risk of oxidation. Therefore, a significant challenge is still posed for fast preparation of MXene film through vacuum-assisted filtration.

We notice that water can pass through the filter membrane quickly during filtration of normal silt. This is because the particle size of silt is larger, and the big gaps between particles and the irregular structure of particles facilitate quick and continual passing of water molecules. Nevertheless, due to the weak interaction between particles, the filter cake will turn into powders easily after filtration and drying. Therefore, conventional cognition tells us that matters that can be filtered within a relatively short time will be in the form of powder instead of film, while MXene assembled layer-by-layer over a relatively long time can form the flexible freestanding film. Obviously, the filter modes for 2D MXene flakes is different to that for three-dimensional (3D) silts. Inspired by silts filtration, and through combination with relatively stronger interaction between the monolayer 2D material flakes, transforming separated and scattered single-layer MXene into silt-like 3D microgel structures may completely settle the problem of slow filtration of MXene dispersion and effectively avoid powdering.

Herein, we propose using ions to induce MXene dispersion microgelation and effectively realize fast preparation of MXene film through combination with vacuum-assisted filtration. The continuous gaps between MXene microgel ensures fast and continual passing of water molecules, reducing filtration time from several hours to dozens of seconds, which is over 1000 times faster. Meanwhile, the strong gelation and riveting effect inside microgel endow this MXene film (named as modified MXene film, MMF) with excellent formability and mechanical properties, allowing the MMF to be freely rubbed and folded. The important thing is that despite the existence of slight nanopore between the MMF layers, the new MMF has still excellent mechanical properties and retains the conductivity of conventional MXene film. Therefore, MMF can replace conventional MXene film in many fields. It must be said that though there are reports on the treatment of MXene by using cations, ${ }^{[22-25]}$ they mainly concentrated on the application of those MXene in energy storage or the interaction between them, the interfacial assembly behavior after the gelation of MXene, the speed-up of vacuum-assisted filtration in particular, has been still not focused on, which will be investigated systematically in this work.

\section{Materials and methods \\ 2.1 Synthesis of MXene $\left(\mathrm{Ti}_{3} \mathrm{C}_{2} \mathrm{~T}_{x}\right)$ dispersion}

$\mathrm{Ti}_{3} \mathrm{C}_{2} \mathrm{~T}_{x}$ MXene was prepared via the etch of the $\mathrm{Ti}_{3} \mathrm{AlC}_{2}$ powders with mixture of $\mathrm{HCl} / \mathrm{LiF}$ based upon the improved minimally intensive layer delamination (MILD) method. ${ }^{[26]}$ Briefly, $40 \mathrm{~mL}$ of $9 \mathrm{M} \mathrm{HCl}$ (Sinopharm Chemical Reagent Company) and $3.2 \mathrm{~g}$ of $\mathrm{LiF}$ (Aladin) were mixed with magnetic stirring in a Teflon beaker. Then, $2 \mathrm{~g}$ of $\mathrm{Ti}_{3} \mathrm{AlC}_{2}$ powders were gradually added to the above mixture and the reaction was allowed for $24 \mathrm{~h}$ under stirring. The resultant was repeatedly washed with deionized water and centrifuged until the $\mathrm{pH}$ of supernatant reached about 6. Afterward, the precipitate was added to $200 \mathrm{~mL}$ of deionized water and appropriate hand shaking was performed. Finally, the dispersion was centrifuged at $3500 \mathrm{rpm}$ for $1 \mathrm{~h}$, and the homogeneous monolayer MXene dispersion (the supernatant liquid) was finally collected.

\subsection{Fabrication of the conventional MXene film}

The conventional MXene film was prepared by filtering $30 \mathrm{~mL}$ of MXene dispersion on a cellulose filter membrane $(0.45 \mu \mathrm{m}$ pore size) using vacuum-assisted filtration, followed by being peeled off carefully from filter membrane after air drying under room temperature. The mass $(30 \mathrm{mg}, 60 \mathrm{mg}$, and 120 $\mathrm{mg}$ ) of conventional MXene film was tuned by adjusting the concentrations $\left(1,2,4 \mathrm{mg} \mathrm{mL}^{-1}\right)$ of the filtered dispersion.

\subsection{Fabrication of the modified MXene film (MMF)}

First, MXene dispersion was mixed with $\mathrm{KOH}$ (Aladin) to form the MXene microgels. The MMF was then prepared by filtering $30 \mathrm{~mL}$ of MXene dispersion containing MXene microgels on a cellulose filter membrane using vacuumassisted filtration, followed by being peeled off carefully from filter membrane after air drying under room temperature. When the mass of MMF is $30 \mathrm{mg}$, the $\mathrm{KOH}$ usages are $0.17 \mathrm{~g}$, $0.25 \mathrm{~g}, 0.40 \mathrm{~g}$ and $0.55 \mathrm{~g}$. When the mass of MMF is $60 \mathrm{mg}$ and $120 \mathrm{mg}$, the $\mathrm{KOH}$ usage is $0.25 \mathrm{~g}$. The MMF induced by other electrolytes such as $\mathrm{NaOH}$ (Aladin), $\mathrm{NaCl}$ (Aladin), $\mathrm{MgCl}_{2}$ (Aladin), $\mathrm{Al}_{2}\left(\mathrm{SO}_{4}\right)_{3}$ (Aladin) and $\mathrm{HCl}$ (Aladin) have the same mass of $30 \mathrm{mg}$ with all electrolyte's usages of $0.25 \mathrm{~g}$. When $0.25 \mathrm{~g} \mathrm{NH}_{3} \cdot \mathrm{H}_{2} \mathrm{O}$ and $\mathrm{CH}_{3} \mathrm{COOH}$ were added into $30 \mathrm{~mL}$ of MXene dispersion, no obvious flocculation was observed and no MMF was prepared.

\subsection{Characterization methods}

Scanning electron microscopy (Hitachi SU800SEM), highangle-annular-darkfield scanning transmission electron microscopy (HAADF-STEM, Talos F200x, FEI), transmission electron microscopy (Tecnai G2 F30, FEI) and atomic force microscopy (Dimension Fastscan, Bruker) were employed to investigate the microstructural morphologies of MXene samples. X-ray diffraction (XRD) data were collected on an X-ray diffractometer (D8 Advance, Bruker) with $\mathrm{Cu} \mathrm{K \alpha}$ radiation $(\lambda=1.5418 \AA)$. Raman spectrum data were collected by HR800 Raman laser (Jobin Yvon Co.). X-ray photoelectron spectroscopy was examined using a Thermo Fisher Scientific ESCALAB $250 \mathrm{Xi}$ XPS. Mechanical properties of film and electrical conductivities were measured with an Instron 3365 universal testing machine, and a 4-ProbeTech RTS-8 four 


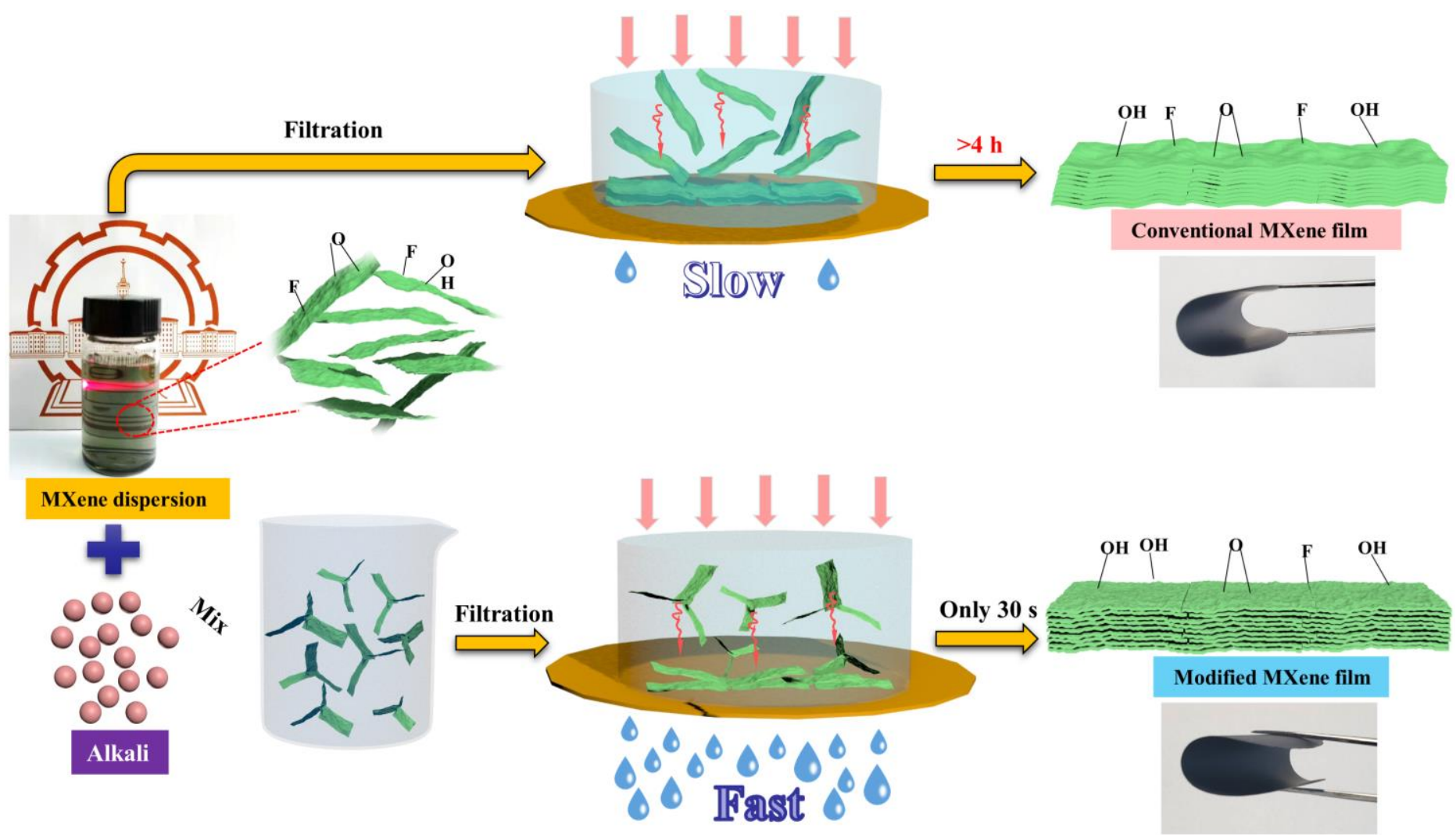

Fig. 1 Schematic of the preparation process of modified MXene film (MMF) via a novel method combining ion induced gelation of MXene dispersion with vacuum-assisted filtration.

probe resistivity meter.

\subsection{Electromagnetic interference shielding measurements} Electromagnetic interference shielding performance of conventional MXene film and MMF samples were examined by an Agilent N5234A vector network analyzer (Keysight Technologies) via wave-guide method in X-band frequency range $(8.2-12.4 \mathrm{GHz})$.

\subsection{Electrochemical measurements}

Cyclic voltammetry (CV), constant current charge/discharge and electrochemical impedance spectroscopy (EIS) were first performed using a CHI 760D workstation (Shanghai Chenhua) with a three-electrode system in $3 \mathrm{M} \mathrm{H}_{2} \mathrm{SO}_{4}$ aqueous electrolyte, in which the prepared free-standing and flexible conventional MXene film and MMF directly served as the working electrode. Meanwhile, $\mathrm{Pt}$ foil and $\mathrm{Ag} / \mathrm{AgCl}$ were used as the counter and reference electrodes, respectively. The constant current charge/discharge and $\mathrm{CV}$ measurements were carried out between -0.5 and $0.1 \mathrm{~V}$. Furthermore, the EIS was measured from $10 \mathrm{MHz}$ to $100 \mathrm{MHz}$ with an amplitude of 5 $\mathrm{mV}$. The gravimetric and volumetric capacitance were calculated using the following formulas:

$$
\begin{gathered}
C_{w t}=\left(\int i d V\right) / \Delta V m v \\
C_{v o l}=\rho \times C_{\mathrm{S}}
\end{gathered}
$$

where $C_{\mathrm{wt}}\left(\mathrm{F} \mathrm{g}^{-1}\right)$ is the gravimetric capacitance, $C_{\mathrm{vol}}\left(\mathrm{F} \mathrm{cm}^{-3}\right)$ is the volumetric capacitance, $i$ is the current density, $v$ is the potential scan rate, $m(\mathrm{~g})$ is the mass of the active material, $V$ is the potential window in the charge-discharge curve, and $\rho$ $\left(\mathrm{g} \mathrm{cm}^{-3}\right)$ is the density of the electrode active material. The density of the electrode material was tested using the formula $\rho=m / d s$, where $s\left(\mathrm{~cm}^{2}\right)$ is the area of the film, $d(\mathrm{~cm})$ is the average thickness of the film detected via SEM, and $m$ (g) is the mass of the film.

\section{Results and discussion}

\subsection{Fabrication and characterization of MMF}

The preparation process of MMF is schematically illustrated in Fig. 1. First, a compound of $\mathrm{HCl}$ and $\mathrm{LiF}$ is used to etch and remove the $\mathrm{Al}$ layer in $\mathrm{Ti}_{3} \mathrm{AlC}_{2}$ to obtain monolayer MXene colloidal dispersion (Fig. S1). Afterward, ions are added into the MXene dispersion to induce flocculate and form a microgel. Then, the fluid containing the microgel is directly placed on filter membrane for vacuum filtration, and MMF can be obtained through room temperature drying. It is important to note that we focus on studying the effect of $\mathrm{KOH}$ addition on MXene dispersion, because the detrimental $-\mathrm{F}$ on MXene surface can be converted into $-\mathrm{OH}$ under alkaline environment, which is very favorable for improving electrochemical properties. ${ }^{[27,28]}$ As shown in Fig. 1, diluted MXene dispersion is light green with a distinct Tyndall effect, suggesting its typical colloid property.

Additionally, the lateral size of $\mathrm{MXene}$ flakes can reach a micrometer scale (Fig. 2a), and such flakes have smooth surface and no obvious traces of oxidation. Meanwhile, the distribution of corresponding Ti element (Fig. 2b) is uniform and obvious, and the corresponding refraction of the selected area shows obvious hexagonal lattice characteristics (Fig. 2c). 


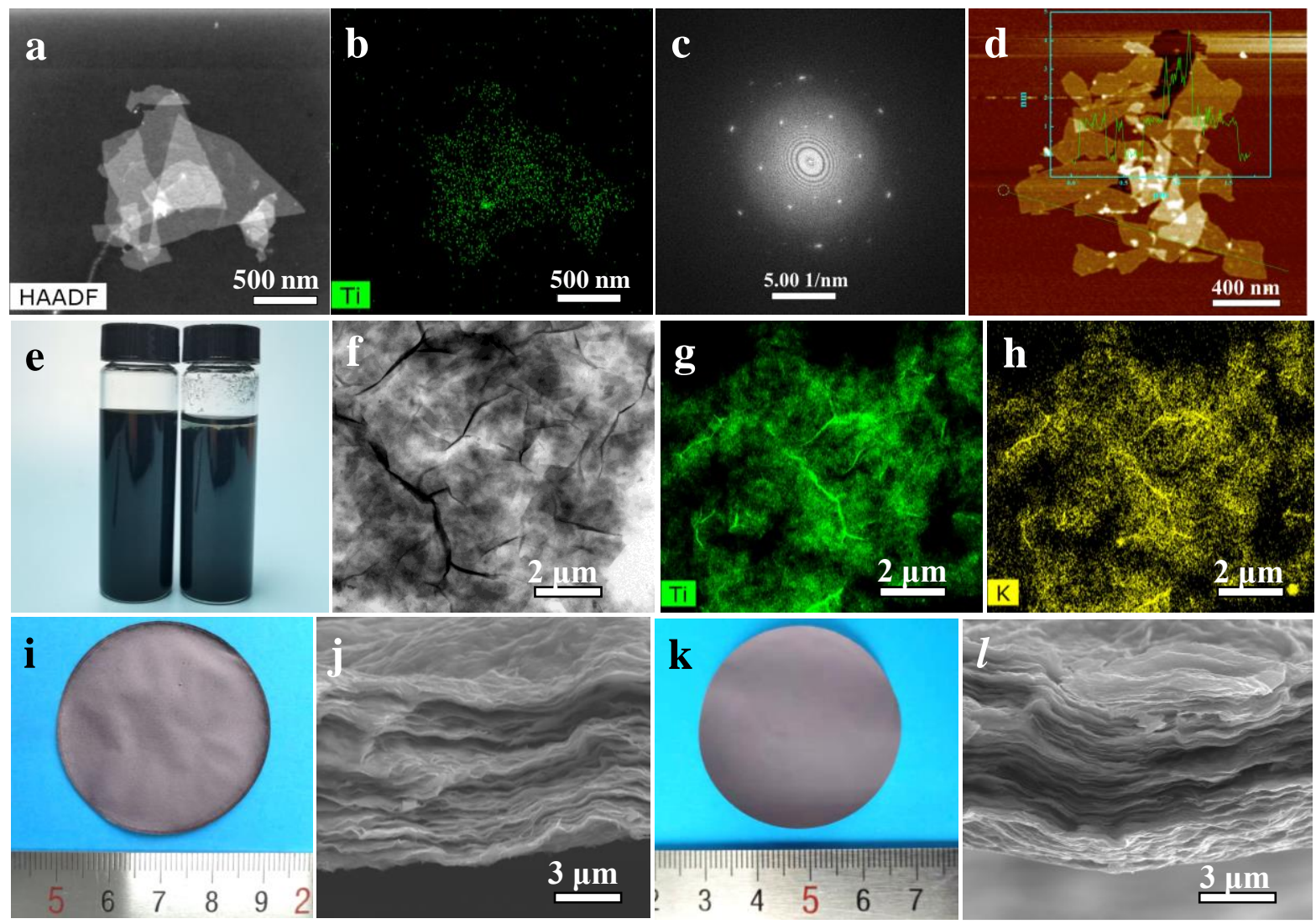

Fig. 2 (a) HAADF-STEM image and (b) EDX elemental mapping of Ti for $\mathrm{Ti}_{3} \mathrm{C}_{2} \mathrm{~T}_{x}$ flakes. (c) Corresponding refraction of the selected area of $\mathrm{Ti}_{3} \mathrm{C}_{2} \mathrm{~T}_{x}$ flakes. (d) $\mathrm{AFM}$ image of $\mathrm{Ti}_{3} \mathrm{C}_{2} \mathrm{~T}_{x}$ flakes. (e) Photographs of $\mathrm{Ti}_{3} \mathrm{C}_{2} \mathrm{~T}_{x}$ dispersion before (left)/after (right) gelation induced by KOH. (f) HAADF-STEM image and EDX elemental mappings of (g) Ti and (h) $\mathrm{K}_{\text {for }} \mathrm{Ti}_{3} \mathrm{C}_{2} \mathrm{~T}_{x}$ microgels. (i) Photograph and (j) Cross-sectional SEM image of the MMF. (k) Photograph and (l) Cross-sectional SEM image of the conventional MXene film.

The atomic force microscopy (AFM) image (Fig. 2d) test result indicates that the thickness of MXene flakes is about 1.3 $\mathrm{nm},{ }^{[29]}$ which is almost their theoretical value. ${ }^{[30,31]}$ This is of great significance to further explore the superior inherent properties of MXene. After $\mathrm{KOH}$ is added, the flocculation phenomenon appears in the stable MXene colloidal dispersion (Fig. 2e, right), and then a 3D microgel structure forms. Fig. $2 \mathrm{f}$ is the HAADF-STEM image of MXene microgel. Unlike the flat structures of MXene flakes, the MXene microgel has a 3D structure consisting of crumpled stacked MXene flakes, with its particle size much bigger than that of MXene flakes. Besides, the AFM image of MXene microgel (Fig. S2) further indicates its 3D crosslinked structure. Fig. $2 \mathrm{~g}$, h and Fig. S3 are the corresponding element distributions of Fig. 2f. The Fig. $2 \mathrm{~h}$ shows uniform distribution of the $\mathrm{K}$ elements, which is caused by cross-linking between $\mathrm{K}^{+}$and MXene flakes. The appearance of MMF (Fig. 2i) is similar to that of conventional MXene film (Fig. 2k), with both having purple surfaces and excellent flexibility (Fig. 1). Note that the cross-sectional scanning electron microscopy (SEM) image of MMF displays a compact structure with residual nanopore between the MMF layers (Fig. 2j), similar with that of compact structure (Fig. 2l) in conventional MXene film. Moreover, as a result of the intercalation effect of $\mathrm{K}^{+}$, the $d$-spacing of MXene film is slight increased (Fig. S4). Raman's result (Fig. S5) shows that the chemical compositions of MMF and conventional MXene film are extremely similar, but the $\mathrm{F}$ element content on MXene surfaces drops from $12 \%$ to $6 \%$ (Fig. S6), induced by $\mathrm{KOH}$. This helps the improvement of electrochemical properties, because the presence of the $-\mathrm{F}$ group hinders diffusion of electrolyte ions ${ }^{[32]}$ In addition, the high resolution of X-ray photoelectron spectroscopy (XPS, Fig. S7) and energy dispersive spectrometer (EDS, Fig. S8) also verify that the $\mathrm{F}$ element is partially removed. It is worth noting that the $\mathrm{K}$ content in MMF is only 1.79 at $\%$ (Fig. S8), thus there is little influence on the essential properties of MXene film.

Conventional preparation of MXene film through vacuumassisted filtration is very slow (Video S1). For instance, it takes $15060 \mathrm{~s}$ (Fig. 3a) to filter all visible water in $30 \mathrm{~mL}$ of 1 $\mathrm{mg} \mathrm{mL}^{-1}$ MXene dispersion. After the addition of $0.25 \mathrm{~g} \mathrm{KOH}$, the filtration time is dramatically shortened to $36 \mathrm{~s}$, and when $0.55 \mathrm{~g}$ of $\mathrm{KOH}$ is added, the rate increases by over 1000 times. This is the presently known fastest preparation rate for MXene film. We make a video to demonstrate the process of preparing MMF (Video S2). It can be noticed that the visible water in MXene microgel dispersion is quickly removed within dozens of seconds after pouring the dispersion into the filter device. Importantly, the MXene microgel can evenly spread over the 

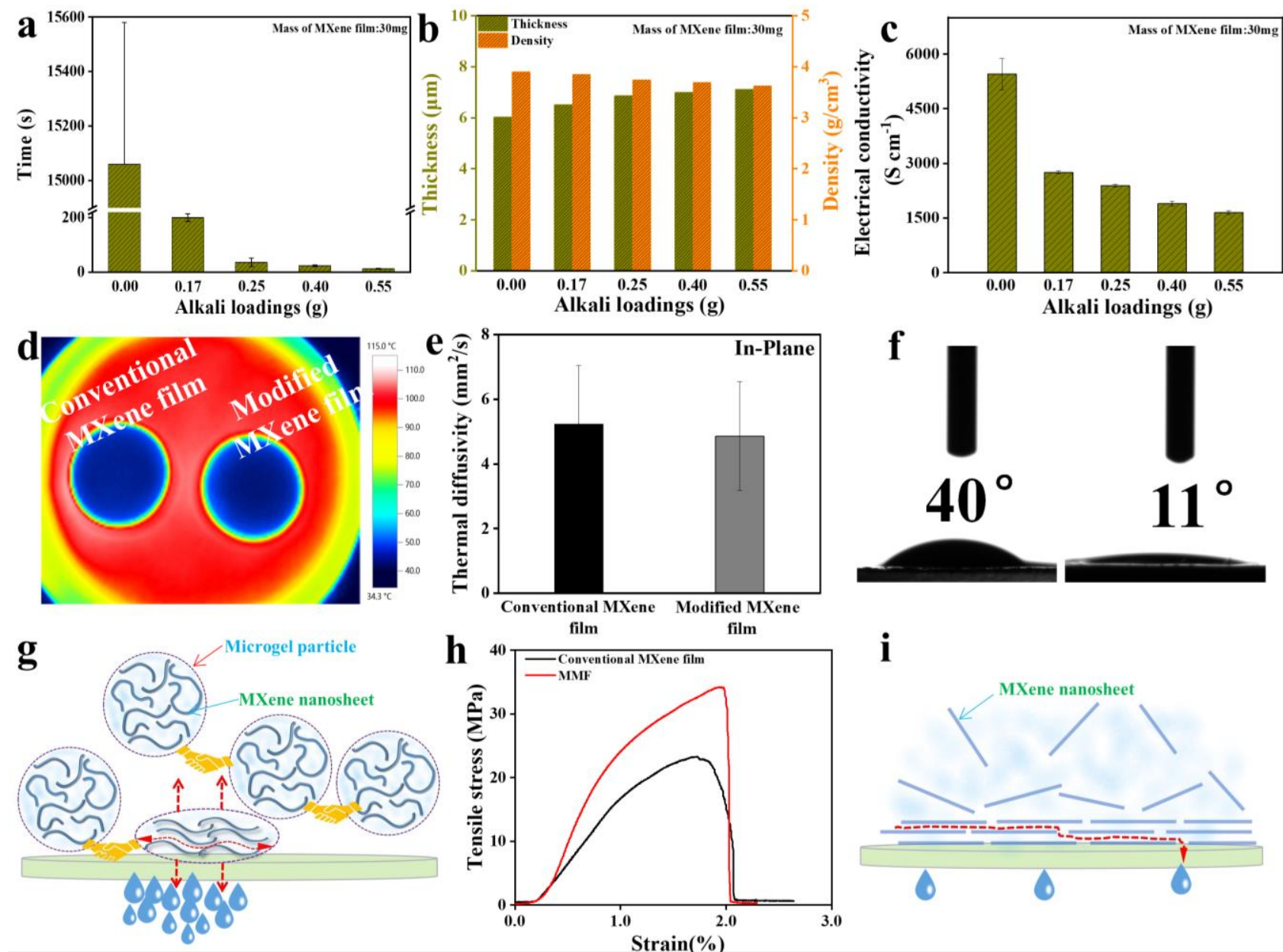

Fig. 3 (a) Filtration time of the conventional MXene film and MMF samples. (b) Thickness and densities of the conventional MXene film and MMF samples. (c) Electrical conductivities of the conventional MXene film and MMF samples. (d) Temperature distribution of the conventional MXene film and MMF on heating stage. (e) In-plane thermal diffusivities of the conventional MXene film and MMF. (f) Contact angles of the conventional MXene film (left) and MMF (right). (g) Possible filtration mechanism of $\mathrm{Ti}_{3} \mathrm{C}_{2} \mathrm{~T}_{x}$ microgels. (h) Static stress-strain curves of the conventional MXene film and MMF. (i) Possible filtration mechanism of $\mathrm{Ti}_{3} \mathrm{C}_{2} \mathrm{~T}_{x}$ flakes.

filter membrane surface within such a short filtration time and be easily separated from the filter membrane surface after drying. Even, we can use our finger to peel the MMF off from the filter membrane rapidly and completely, indicating the outstanding mechanical property of MMF. More interestingly, MMF can always maintain its structural integrity even be suffered arbitrary folding and repeated rub, exhibiting the excellent flexibility. Due to the similar compact structure (Fig. $2 \mathrm{j}$ ), the density of MMF is only descend slightly compared with conventional MXene film because of the existence of slight nanopore (Fig. 3b). Note that the electrical conductivity of the MMF will decrease with the increase of the addition of $\mathrm{KOH}$ (Fig. 3c). The drop might be due to the slight increase of $d$-spacing, the formation of slight nanopore and the remaining insulated $\mathrm{KOH}$. With a comprehensive consideration of preparation efficiency and electrical conductivity, we give priority to the MMF sample with the addition of $0.25 \mathrm{~g} \mathrm{KOH}$, whose electrical conductivity is $2385 \mathrm{~S} \mathrm{~cm}^{-1}$. It needs to be noted that the thicker and greater the mass loading of the MXene film, the greater its application value in fields such as electrochemical energy storage and other fields. ${ }^{[9,27,33]}$ However, it is vastly difficult to prepare thick MXene film through conventional vacuum-assisted filtration due to the need of more time and energy. The time required for preparing $60 \mathrm{mg}$ and $120 \mathrm{mg}$ of MMF with the method in this work is only $61 \mathrm{~s}$ and $170 \mathrm{~s}$, respectively (Fig. S9). In contrast, it takes over 10 hours to prepare $120 \mathrm{mg}$ of MXene film through the conventional filtration method. With only one filtration device, we can easily prepare a large amount of MXene film within 1 hour on a large scale (Fig. S10), and we can even prepare 225 mg MXene film within a very short time (Fig. S11), which is of great significance to promote large-scale application of MXene film. The introduction of $\mathrm{KOH}$ has little influence on capacity of cross plane thermal insulation of MXene film. As shown in Fig. 3d, the thermographs of MMF and conventional MXene film on the heating platform are practically the same, implying that they have the similar capacity of cross plane thermal insulation, and this conclusion is further proven by the thermal diffusion coefficient test result (Fig. 3e). More interestingly, introducing $\mathrm{KOH}$ can also decrease the contact 
angle of MXene film (Fig. 3f), which is also favorable for improving the wettability and electrochemical performance of MXene film. Besides, MMF can be also used as an actuator based on moisture gradients like conventional MXene film (Fig. S12). ${ }^{[13]}$

\subsection{Formation and the filtration mechanism of MXene microgel}

To investigate the formation and the filtration mechanism of MXene microgel, $\zeta$ potential test characterization is performed. The pristine MXene dispersion has outstanding colloidal stability and the colloidal particle is negatively charged with the $\zeta$ potential of $-54 \mathrm{mV}$. The introduction of $\mathrm{KOH}$ decreases the charge $(\zeta$ potential shifts toward positive direction, Fig. S13a) and the thickness of diffusion layer. Therefore, flocculation of the MXene dispersion quickly occurs and crumpled 3D microgel structures forms, increasing the average size of particle (Fig. S13b). In microgel structures, it is possible that the MXene flakes mainly interact with each other in a surface-to-surface manner ${ }^{[25]}$ and water molecules can pass through rapidly in the interconnected structure. The specific possible filtration mechanism is shown in Fig. $3 \mathrm{~g}$. MXene microgel particles will accumulate at the interface in a highly ordered manner once filtration starts. Subsequently, water molecules will pass through the space of the microgel continual network structure immediately. Besides, the curly structures of MXene flakes will retain a large amount of continuous nanopore channels during the microgel accumulation for water molecules to rapidly pass through and therefore ensures continuous water drainage. Meanwhile, the 3D structures of the MXene microgel will gradually collapse under vacuum negative pressure to make the MXene microgel densify and tightly accumulate as water drains, which causes the closure of most of nanopore and the high density of MMF. While strong interaction force between MXene microgel particles makes the MXene microgel particles tightly embed together to form a complete film structure. Besides, $\mathrm{K}^{+}$also plays a role in cross-linking and enhancement between MXene layers (Fig. 3h), which offsets bad effect of the existence of slight nanopore on mechanical property. In comparison, when the conventional MXene colloidal dispersion is filtered (Fig. 3i), a small number of MXene flakes will rapidly deposit on the surface of filter membrane in a surface-to-surface manner and form MXene layers of parallel arranged compact MXene film. After that, a large number of MXene flakes remaining in the colloidal dispersion will gradually stack layer by layer on the formed MXene film surface. Due to the high aspect ratio and barrier effect of the MXene flakes, drained water needs to slowly go around edges of the MXene flakes and the transportation paths are serpentine, causing low preparation efficiency. Accordingly, transforming rambling MXene flakes into 3D MXene microgel is the premise for enhancing preparation efficiency. It is noteworthy that weak electrolyte with extremely weak gelation induction ability, such as $\mathrm{NH}_{3} \cdot \mathrm{H}_{2} \mathrm{O}$ and $\mathrm{CH}_{3} \mathrm{COOH}$, almost cannot convert MXene dispersion into 3D MXene microgel structure. Other strong electrolytes, such as $\mathrm{NaOH}, \mathrm{NaCl}, \mathrm{MgCl}_{2}, \mathrm{HCl}$ and $\mathrm{Al}_{2}\left(\mathrm{SO}_{4}\right)_{3}$ can accelerate flocculation of dispersion (Fig. S14) and also effectively accelerate the MXene film filtration rate (Fig. $\mathrm{S} 15,16)$. The electrical conductivities of MMF are not always reduced like that of MMF treated with $\mathrm{KOH}$, which will be affected by the kinds of electrolytes (Fig. S17). For instance, $\mathrm{Al}_{2}\left(\mathrm{SO}_{4}\right)_{3}$ can not only reduce filtration time significantly but also maintain electrical conductivity to a great extent $(97 \%$ of conventional MXene film), while $\mathrm{HCl}$ can even help the improvement of electrical conductivity. Therefore, an optimal combination of properties, such as filtration time and electrical conductivity can be achieved by selecting a proper electrolyte based on needs. Remarkably, MXene film cannot be obtained from post-ion induction MXene microgel through drop casting (Fig. S18), which once more proves the uniqueness and superiority of MXene film prepared through the method in this work. Meanwhile, the method in this work can also be extended to the fast preparation of other 2D materials. For example, it takes days or even longer to filter conventional graphene oxide dispersion fluids, while after microgelation, the time will be shortened to around one hour (Fig. S19), improving preparation efficiency.

\subsection{Applications of MMF in supercapacitors and EMI shielding materials}

To date, the applications of $\mathrm{Ti}_{3} \mathrm{C}_{2} \mathrm{~T}_{x}$ and other MXene in energy storage received wide spread researcher coverage. ${ }^{[34-38]}$ To verify the practical application potential of MMF, we investigate its performances as supercapacitor electrode materials. Freestanding MMF can be directly used as working electrode without requiring any additives. Under a low scan rate $\left(2 \mathrm{mV} \mathrm{s}^{-1}\right)$, both MMF (Fig. $\left.4 \mathrm{a}\right)$ and conventional MXene film (Fig. 4b) show unique intercalation pseudocapacitance redox peak of $\mathrm{Ti}_{3} \mathrm{C}_{2} \mathrm{~T}_{x}$ in acid electrolyte. ${ }^{[9,39]}$ However, the cyclic voltammetry $(\mathrm{CV})$ curve integral area of MMF is obviously larger than that of conventional MXene film, suggesting the superior capacitance in MMF. This is mainly because the $F$ groups on the terminal surface in MMF are significantly fewer than those of conventional MXene film, increasing the proportion of active groups being able to participate in pseudocapacitance reactions. ${ }^{[27,28]} \mathrm{Fig} .4 \mathrm{c}$ is the charge-discharge curves of MMF at various current densities, and it is clear that the sample has wide humps typical of $\mathrm{Ti}_{3} \mathrm{C}_{2} \mathrm{~T}_{x}$ and a charge-discharge curve close to a triangle. Fig. $4 \mathrm{~d}$ is the corresponding specific capacitance performances of MMF and conventional MXene film at various scan rates. The specific capacitance of MMF $(0.25 \mathrm{~g} \mathrm{KOH})$ at $2 \mathrm{mV} \mathrm{s}^{-1}$ is 540 $\mathrm{F} \mathrm{g}^{-1}$, significantly higher than that of conventional MXene film $\left(350 \mathrm{~F} \mathrm{~g}^{-1}\right)$ and other electrode materials; ${ }^{[40,41]}$ togethering with high density of MMF, the volumetric capacitance can reach up to $2020 \mathrm{~F} \mathrm{~cm}^{-3}$, which is one of the highest values among the pure MXene film. $\cdot^{[8-10,27,42]}$ Additionally, compared with conventional MXene film, MMF has lower interface charge transfer resistance (Fig. $4 \mathrm{e}$ ) and better long-term 

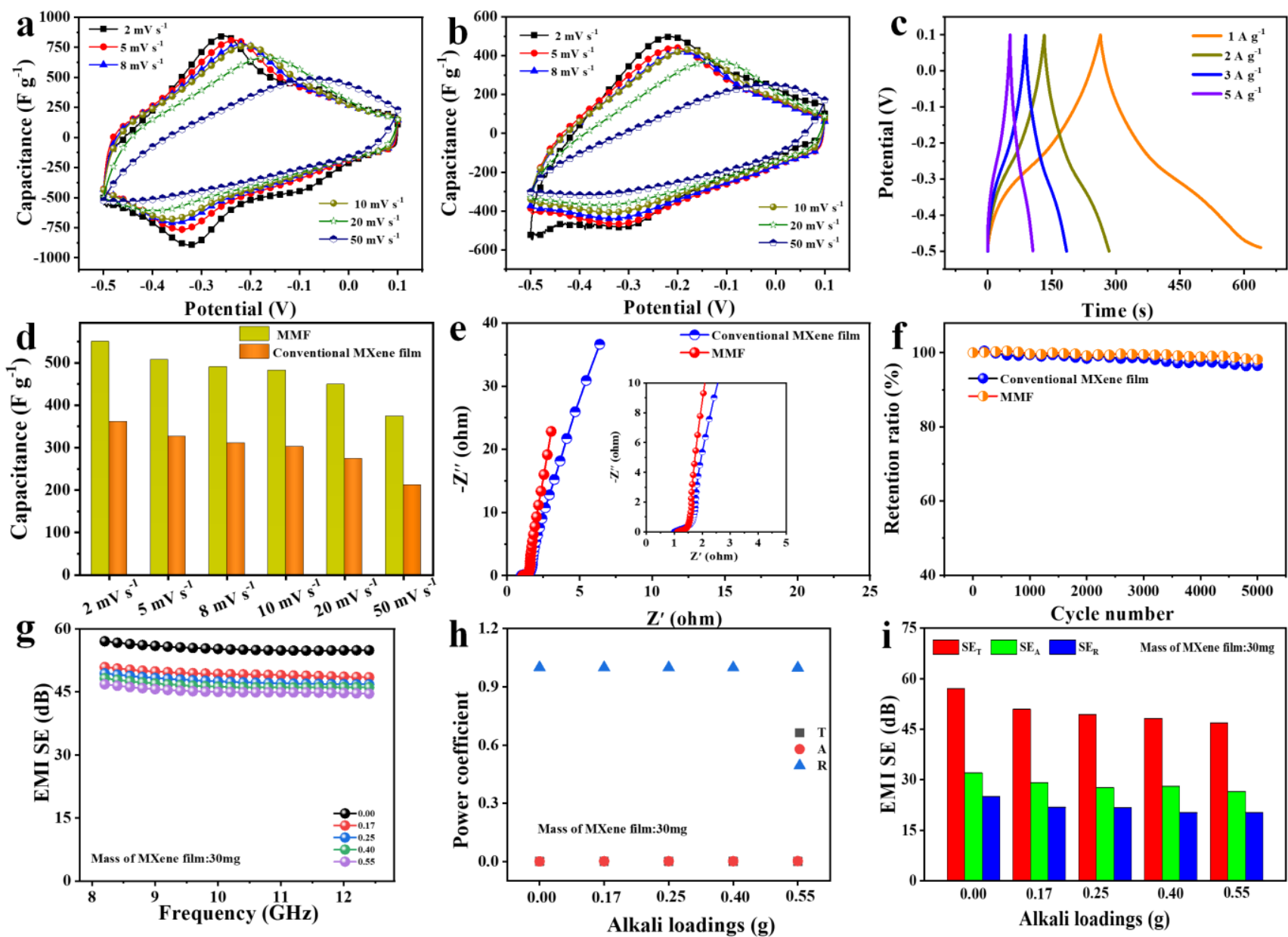

Fig. 4 (a) CV curves of the MMF at the various scan rates. (b) CV curves of the conventional MXene film at the various scan rates. (c) Constant current charge-discharge curves of the MMF at various current densities. (d) Gravimetric capacitances of the conventional MXene film and MMF at different scan rates. (e) Nyquist plots of the conventional MXene film and MMF. (f) Capacitance retention of the conventional MXene film and MMF at a current density of $5 \mathrm{~A} \mathrm{~g}^{-1}$ for 5000 cycles. (g) EMI SE of the conventional MXene film and MMF samples. (h) $T, A$ and $R$ values of the conventional MXene film and MMF samples at $8.2 \mathrm{GHz}$. (i) The comparison of $\mathrm{SE}_{\mathrm{T}}, \mathrm{SE}_{\mathrm{A}}$ and $\mathrm{SE}_{\mathrm{R}}$ of the conventional MXene film and MMF samples at $8.2 \mathrm{GHz}$.

cycling durability (Fig. 4f). Therefore, MMF shows more excellent electrochemical performance. To further explore the electrochemical capacitance behavior of MMF, rate capability test is conducted (Fig. 4a, b). It shows that the CV curves of MMF and conventional MXene film present similar trend with the increase of scan rates, indicating that they have similar rate capability. Note that the enlarged $d$-spacing and the existence of slight nanopore should have enabled MMF to exhibit more enhanced rate capability relative to conventional MXene film, but it is not supported by the experiment data. The results further proved the collapse of pore structures and closure of most of them, agreeing with the high density and compact structure of MMF, and the capillary compression generated by the pre-hydrogel membrane during water evaporation might be also contribute to it. But it needs to be emphasized that the study of electrochemical properties is not the main purpose in this paper, we mainly focus on the proposition of a new method to accelerate the preparation of MXene film.

Although the electrical conductivity of MMF is lower than conventional MXene film, it still gives outstanding EMI shielding effectiveness (SE). As shown in Fig. 4g, both MMF and conventional MXene film exhibit weak dependence on frequency over the whole X-band. The EMI SE of MMF $(0.25$ $\mathrm{g} \mathrm{KOH})$ is $49 \mathrm{~dB}$, which is far more than the standard minimal shielding requirement in practical application $(>20 \mathrm{~dB})$. When the thickness of MMF is $36.7 \mu \mathrm{m}(120 \mathrm{mg})$, its EMI SE can reach $66 \mathrm{~dB}$ (Fig. S20a); when two pieces of MMF (120 mg) are stacked, the EMI SE is as high as $94 \mathrm{~dB}$, even higher than that of conventional MXene film with mass of $120 \mathrm{mg}$ ( $85 \mathrm{~dB}$ ) (Fig. S20b), which means more than $99.99999996 \%$ incident radiation can be blocked. Besides, the absolute EMI SE (SSE/t, specific EMI SE divided by thickness) value of MMF is 19333 $\mathrm{dB} \cdot \mathrm{cm}^{2} \cdot \mathrm{g}^{-1}$ (Fig. S21a), which is higher than most of the other reported EMI shielding materials. ${ }^{[43-48]}$ The SSE/ $t$ values of other MMF samples are shown in Fig. S21b, c. It is noteworthy that the reflection coefficient $(R)$ in all samples are larger than transmission coefficient $(T)$ and absorption coefficient $(A)$ (Fig. 4h). In addition, $R$ is very close to 1 and $T$ is very small, suggesting that the decisive shielding mechanism of MMF is reflection. The increase of $T$ and $A$ and the drop of $R$ will 
happen with the increase of $\mathrm{KOH}$ usage (Fig. S22). The greater the MMF thickness, the greater the $R$ value and the smaller $T$ and $A$ values (Fig. S23). Furthermore, microwave absorption $\left(\mathrm{SE}_{\mathrm{A}}\right)$ of MMF is higher than the microwave reflection $\left(\mathrm{SE}_{\mathrm{R}}\right)$, which is similar to conventional MXene film (Fig. 4i). It should be noted that the electromagnetic wave reflection occurs before absorption, thus $\mathrm{SE}_{\mathrm{A}}$ means the capacity of material to attenuation the electromagnetic waves of transmitted into material. ${ }^{[49]}$ Therefore, lower $\mathrm{SE}_{\mathrm{R}}$ and larger $\mathrm{SE}_{\mathrm{A}}$ suggest that attenuated electromagnetic waves that transmitted into MMF are more caused by absorption than by reflection. Moreover, the addition of $\mathrm{KOH}$ will slightly reduce the $\mathrm{SE}_{\mathrm{R}}$ and $\mathrm{SE}_{\mathrm{A}}$ (Fig. 4i) while increase of thickness will increase $\mathrm{SE}_{\mathrm{A}}$ but has little effect on $\mathrm{SE}_{\mathrm{R}}$ (Fig. S24). The possible electromagnetic waves attenuation mechanism of MMF is shown in Fig. S25. When incident electromagnetic waves reach high-conductivity MMF surface, a portion of electromagnetic waves will be immediately reflected. When the rest electromagnetic waves that enter the MMF pass through MXene lattices, they interact with high-charge density of MXene to yield electricity, causing the ohm loss and electromagnetic wave energy reduction. Moreover, the nanopore structures between MMF layers facilitate multireflection of electromagnetic waves, which enables the electromagnetic waves to be further dissipated and absorbed. There are a certain number of oxygen-containing functional groups and residual fluorine groups on the MMF surface; dipolar polarization may occur between $\mathrm{Ti}$ in the electromagnetic field and these functional groups, resulting in polarization loss. ${ }^{[11,50]}$ Therefore, MMF is an excellent EMI shielding material for electromagnetic wave interference management.

\section{Conclusions}

Inspired by filtration of silt, a fast MXene film preparation method combining ion-induced MXene dispersion gelation and vacuum-assisted filtration is proposed. This method can reduce the preparation time from several hours to dozens of seconds, being the fastest existing method. The key to fast preparation is proper gaps between MXene microgel, strong gelation and riveting effect inside microgel. MMF features excellent mechanical properties and being able to be folded and rubbed, and maintains extraordinary conductivity while containing slight nanopores, showing more enhanced electrochemical properties relative to conventional MXene film with the volumetric capacitance reaching $2020 \mathrm{~F} \mathrm{~cm}^{-3}$, which is one of the greatest values for pure MXene film; with a thickness of $36.7 \mu \mathrm{m}$, EMI shielding effectiveness value can reach up to $66 \mathrm{~dB}$. This work provides important theoretical and technical support for fast preparation of MXene film with excellent comprehensive properties, which is significantly important for the large-scale preparation and application of MXene film.

\section{Acknowledgements}

This work was supported by the National Natural Science Foundation of China (52002095), China Postdoctoral Science Foundation (2019M661274) and Heilongjiang Postdoctoral Fund (LBH-Z19140).

\section{Supporting information}

Applicable

\section{Conflict of interest}

There are no conflicts to declare.

\section{References}

[1] B. Anasori, M. R. Lukatskaya, Y. Gogotsi, Nat. Rev. Mater., 2017, 2, 16098, doi: 10.1038/natrevmats.2016.98.

[2] Y. Gogotsi, B. Anasori, ACS Nano., 2019, 13, 8491, doi: 10.1021/acsnano.9b06394.

[3] Y. Wang, Y. Liu, C. Wang, H. Liu, J. Zhang, J. Lin, J. Fan, T. Ding, J. E. Ryu, Z. Guo, Eng. Sci., 2020, 9, 50, doi: 10.30919/es8d903.

[4] S. A. Mirkhani, A. Shayesteh Zeraati, E. Aliabadian, M. Naguib, U. Sundararaj, ACS Appl. Mater. Interfaces., 2019, 11, 18599, doi: 10.1021/acsami.9b00393.

[5] J. Liu, H.-B. Zhang, R. Sun, Y. Liu, Z. Liu, A. Zhou, Z.-Z. Yu, Adv. Mater., 2017, 29, 1702367, doi: 10.1002/adma.201702367.

[6] H. Yu, Y. Wang, Y. Jing, J. Ma, C.-F. Du, Q. Yan, Small, 2019, 15, 1901503, doi: 10.1002/smll.201901503.

[7] C. E. Shuck, A. Sarycheva, M. Anayee, A. Levitt, Y. Zhu, S. Uzun, V. Balitskiy, V. Zahorodna, O. Gogotsi, Y. Gogotsi, Adv. Eng. Mater., 2020, 22, 1901241, doi: 10.1002/adem.201901241.

[8] Z. Fan, Y. Wang, Z. Xie, X. Xu, Y. Yuan, Z. Cheng, Y. Liu, Nanoscale, 2018, 10, 9642, doi: 10.1039/C8NR01550C.

[9] M. R. Lukatskaya, S. Kota, Z. Lin, M.-Q. Zhao, N. Shpigel, M. D. Levi, J. Halim, P.-L. Taberna, M. W. Barsoum, P. Simon, Y. Gogotsi, Nat. Energy, 2017, 2, 17105, doi: 10.1038/nenergy.2017.105.

[10] J. Yan, C. E. Ren, K. Maleski, C. B. Hatter, B. Anasori, P. Urbankowski, A. Sarycheva, Y. Gogotsi, Adv. Funct. Mater., 2017, 27, 1701264, doi: 10.1002/adfm.201701264.

[11] F. Shahzad, M. Alhabeb, C. B. Hatter, B. Anasori, S. Man Hong, C. M. Koo, Y. Gogotsi, Science, 2016, 353, 1137, doi: 10.1126/science.aag2421.

[12] H. Chen, Y. Wen, Y. Qi, Q. Zhao, L. Qu, C. Li, Adv. Funct. Mater., 2020, 30, 1906996, doi: 10.1002/adfm.201906996.

[13] J. Wang, Y. Liu, Z. Cheng, Z. Xie, L. Yin, W. Wang, Y. Song, H. Zhang, Y. Wang, Z. Fan, Angew. Chem. Int. Ed., 2020, 59, 14029, doi: 10.1002/anie.202003737.

[14] G. Cai, J.-H. Ciou, Y. Liu, Y. Jiang, P. S. Lee, Sci. Adv., 2019, 5, eaaw7956, doi: 10.1126/sciadv.aaw7956. 
[15] J. Cao, Z. Zhou, Q. Song, K. Chen, G. Su, T. Zhou, Z. Zheng, C. Lu, X. Zhang, ACS Nano., 2020, 14, 7055, doi: 10.1021/acsnano.0c01779.

[16] J. Wang, H. Ma, Y. Liu, Z. Xie, Z. Fan, ChemPlusChem, 2021, 86, 406, doi: 10.1002/cplu.202000828.

[17] R. Li, L. Zhang, L. Shi, P. Wang, ACS Nano., 2017, 11, 3752, doi: 10.1021/acsnano.6b08415.

[18] L. Ding, Y. Wei, L. Li, T. Zhang, H. Wang, J. Xue, L.-X. Ding, S. Wang, J. Caro, Y. Gogotsi, Nat. Commun., 2018, 9, 155, doi: 10.1038/s41467-017-02529-6.

[19] Y. Su, V. G. Kravets, S. L. Wong, J. Waters, A. K. Geim, R. R. Nair, Nat. Commun., 2014, 5, 4843, doi:10.1038/ncomms5843.

[20] Y.-H. Yang, L. Bolling, M. A. Priolo, J. C. Grunlan, $A d v$. Mater., 2013, 25, 503, doi: 10.1002/adma.201202951.

[21] N. Nidamanuri, Y. Li, Q. Li, M. Dong, Eng. Sci., 2020, 9, 3, doi: 10.30919/es8d128906.

[22] D. Zhao, M. Clites, G. Ying, S. Kota, J. Wang, V. Natu, X. Wang, E. Pomerantseva, M. Cao, M. W. Barsoum, Chem. Commun., 2018, 54, 4533, doi: 10.1039/C8CC00649K.

[23] A. VahidMohammadi, M. Mojtabavi, N. M. Caffrey, M. Wanunu, M. Beidaghi, Adv. Mater., 2019, 31, 1806931, doi: 10.1002/adma.201806931.

[24] V. Natu, M. Clites, E. Pomerantseva, M. W. Barsoum, Mater. Res. Lett., 2018, 6, 230, doi: 10.1080/21663831.2018.1434249.

[25] M. Alhabeb, K. Maleski, B. Anasori, P. Lelyukh, L. Clark, S. Sin, Y. Gogotsi, Chem. Mater., 2017, 29, 7633, doi: 10.1021/acs.chemmater.7b02847.

[26] Z. Fan, Y. Wang, Z. Xie, D. Wang, Y. Yuan, H. Kang, B. Su, Z. Cheng, Y. Liu, Adv. Sci., 2018, 5, 1800750, doi: 10.1002/advs.201800750.

[27] J. Li, X. Yuan, C. Lin, Y. Yang, L. Xu, X. Du, J. Xie, J. Lin, J. Sun, Adv. Energy Mater., 2017, 7, 1602725, doi: 10.1002/aenm.201602725

[28] Z. Fan, H. He, J. Yu, L. Liu, Y. Liu, Z. Xie, ACS Appl. Energy Mater., 2020, 3, 8171, doi: 10.1021/acsaem.0c01650. [29] A. Lipatov, M. Alhabeb, M. R. Lukatskaya, A. Boson, Y. Gogotsi, A. Sinitskii, Adv. Electron. Mater., 2016, 2, 1600255, doi: 10.1002/aelm.201600255.

[30] Z. Fan, H. He, J. Yu, J. Wang, L. Yin, Z. Cheng, Z. Xie, Y. Wang, Y. Liu, ACS Materials Lett., 2020, 1598, doi: 10.1021/acsmaterialslett.0c00422.

[31] Q. Tang, Z. Zhou, P. Shen, J. Am. Chem. Soc., 2012, 134, 16909, doi: 10.1021/ja308463r.

[32] Y. Xia, T. S. Mathis, M.-Q. Zhao, B. Anasori, A. Dang, Z. Zhou, H. Cho, Y. Gogotsi, S. Yang, Nature, 2018, 557, 409, doi: 10.1038/s41586-018-0109-z.
[33] V. Natu, M. Sokol, L. Verger, M. W. Barsoum, J. Phys. Chem. C, 2018, 122, 27745, doi: 10.1021/acs.jpcc.8b08860.

[34] X. Li, M. Li, Z. Huang, G. Liang, Z. Chen, Q. Yang, Q. Huang, C. Zhi, Energy Environ. Sci., 2021, 14, 407-413, doi: 10.1039/D0EE03086D.

[35] J. Nan, X. Guo, J. Xiao, X. Li, W. Chen, W. Wu, H. Liu, Y. Wang, M. Wu, G. Wang, Small, 2021, 17, 1902085, doi: 10.1002/smll.201902085.

[36] X. Li, M. Li, Q. Yang, D. Wang, L. Ma, G. Liang, Z. Huang, B. Dong, Q. Huang, C. Zhi, Adv. Energy Mater., 2020, 10 (35), 2001394, doi: 10.1002/aenm.202001394.

[37] C. Zhang, L. Cui, S. Abdolhosseinzadeh, J. Heier, InfoMat, 2020, 2, 613, doi: 10.1002/inf2.12080.

[38] X. Li, N. Li, Z. Huang, Z. Chen, G. Liang, Q. Yang, M. Li, Y. Zhao, L. Ma, B. Dong, Q. Huang, J. Fan, C. Zhi, Adv. Mater., 2021, 33 (8), 2006897, doi: 10.1002/adma.202006897. [39] M. Hu, Z. Li, T. Hu, S. Zhu, C. Zhang, X. Wang, ACS Nano., 2016, 10, 11344, doi: 10.1021/acsnano.6b06597.

[40] S. u. Rehman, R. Ahmed, K. Ma, S. Xu, T. Tao, M. A. Aslam, M. Amir, J. Wang, Eng. Sci., 2020, 13, 71, doi: 10.30919/es8d1263.

[41] X. Yang, J. Zhu, L. Qiu, D. Li, Adv. Mater., 2011, 23, 2833, doi: 10.1002/adma.201100261.

[42] C. Yang, Y. Tang, Y. Tian, Y. Luo, Y. He, X. Yin, W. Que, Adv. Funct. Mater., 2018, 28, 1705487, doi: 10.1002/adfm.201705487.

[43] F. Moglie, D. Micheli, S. Laurenzi, M. Marchetti, V. Mariani Primiani, Carbon, 2012, 50, 1972, doi: 10.1016/j.carbon.2011.12.053.

[44] M. Crespo, M. González, A. L. Elías, L. Pulickal Rajukumar, J. Baselga, M. Terrones, J. Pozuelo, Phys. Status Solidi-R, 2014, 8, 698, doi: 10.1002/pssr.201409151.

[45] W.-L. Song, X.-T. Guan, L.-Z. Fan, W.-Q. Cao, C.-Y. Wang, M.-S. Cao, Carbon, 2015, 93, 151, doi: 10.1016/j.carbon.2015.05.033.

[46] B. Shen, Y. Li, D. Yi, W. Zhai, X. Wei, W. Zheng, Carbon, 2016, 102, 154, doi: 10.1016/j.carbon.2016.02.040.

[47] W.-T. Cao, F.-F. Chen, Y.-J. Zhu, Y.-G. Zhang, Y.-Y. Jiang, M.-G. Ma, F. Chen, ACS Nano, 2018, 12, 4583, doi: 10.1021/acsnano.8b00997.

[48] Q. Gao, Y. Pan, G. Zheng, C. Liu, C. Shen, X. Liu, Adv. Compos. Hybrid Mater., 2021, doi: 10.1007/s42114-02100221-4.

[49] Z. Fan, D. Wang, Y. Yuan, Y. Wang, Z. Cheng, Y. Liu, Z. Xie, Chem. Eng. J., 2020, 381, 122696, doi: 10.1016/j.cej.2019.122696. 


\section{Author information}

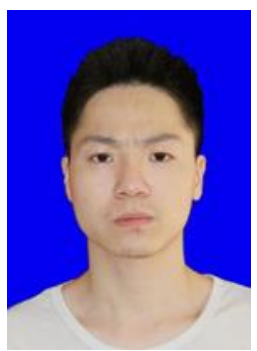

Jingfeng Wang received his Bachelors and Masters both from the Northwest $A \& F$ University, China. Currently, he is a Ph.D. student under the supervision of Professor Yuyan Liu and Assistant Professor Zhimin Fan at Harbin Institute of Technology. His research interests focus on the development and characterization of novel two-dimensional materials in the fields of soft actuators, smart materials, energy storage and electromagnetic interference shielding.

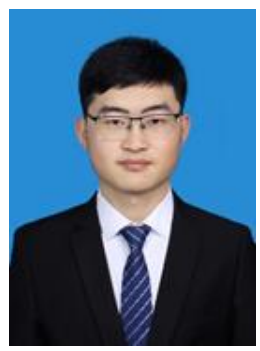

Hui Kang is an undergraduate under the supervision of Professor Yuyan Liu and Assistant Professor Zhimin Fan at Harbin Institute of Technology. His research interests focus on controllable construction of MXene film and its application in electromagnetic interference shielding and wearable devices.

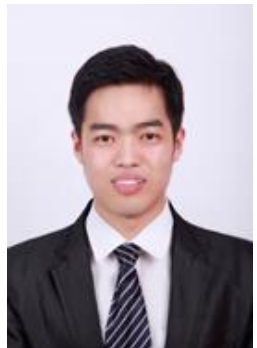

Haoxiang Ma is a research associate at Institute of Deep-sea Science and Engineering, Chinese Academy of Sciences. He received his Ph.D. degree from Harbin Institute of Technology in 2018. Ma's current research interest covers the areas of shape memory polymer, intelligent and functional polymer materials, sensor and electromagnetic interference shielding materials.

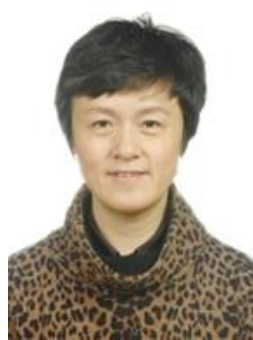

Yuyan Liu is a Professor in the School of Chemistry and Chemical Engineering at Harbin Institute of Technology. Liu's current research interest covers the areas of shape memory polymer, intelligent and functional polymer materials, flexible composites and bio-inspired functional

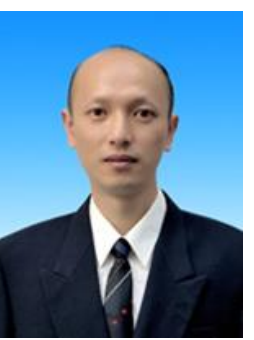

Zhimin Xie is a Professor in the School of Astronautics at Harbin Institute of Technology. Xie's current research interest covers the areas of shape memory polymer, intelligent and functional polymer materials, space inflatable structures and electromagnetic interference shielding materials.

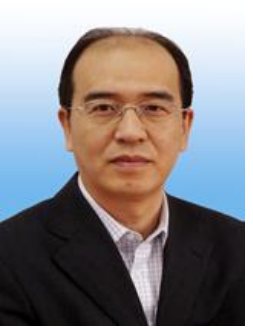

Youshan Wang is a Professor in the School of Astronautics at Harbin Institute of Technology. Wang's current research interest covers the areas of rubber materials, intelligent and functional polymer materials, space inflatable structures and electromagnetic interference shielding materials.

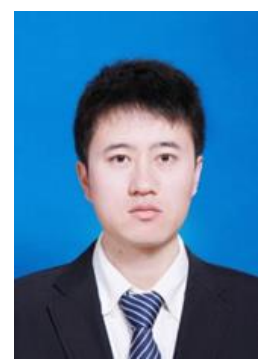

Zhimin Fan is an Assistant Professor at Harbin Institute of Technology. He received his Ph.D. degree from Harbin Institute of Technology in 2019. His research interests focus on the synthesis and application of novel two-dimensional materials in the fields of energy storage, soft actuators, smart materials, and electromagnetic interference shielding.

Publisher's Note: Engineered Science Publisher remains neutral with regard to jurisdictional claims in published maps and institutional affiliations.

surface. 